\title{
RELAÇÃO ENTRE GRAVIDADE DO DESVIO FONOLÓGICO E FATORES FAMILIARES
}

\author{
Relationship among severity of phonological disorders \\ and familial factors
}

\author{
Karina Carlesso Pagliarin (1), Ana Rita Brancalioni (2), Márcia Keske-Soares ${ }^{(3)}$, \\ Ana Paula Ramos de Souza ${ }^{(4)}$
}

\section{RESUMO}

Objetivo: analisar a relação entre os fatores familiares e as diferentes gravidades. Método: foram analisadas entrevistas iniciais, realizadas com base em um protocolo padronizado que incluía questões referentes à história gestacional, a antecedentes patológicos familiares e ao relacionamento familiar de 152 crianças com desvio fonológico, com idades entre 4:0 a 8:0 anos. Os fatores familiares investigados foram: gravidez não planejada; dependência (de um dos pais e/ou ambos) de álcool e/ ou drogas; distúrbios de fala, linguagem, e/ou audição apresentados por pais e/ou familiares de primeiro grau; distúrbios psicológicos apresentados pelos pais; pais separados; pai ausente e perda de parentes próximos. Em seguida, a gravidade do desvio fonológico foi calculada segundo o Percentual de Consoantes Corretas-Revisado e, classificada em quatro grupos: desvio leve $(n=49)$, leve-moderado $(n=67)$, moderado-grave $(n=24)$, grave $(n=12)$. Por fim, os dados foram tabulados e submetidos a tratamento estatístico, utilizando o teste Exato de Fisher, considerando-se $p<0,05$. Resultados: verificou-se um predomínio do grupo com grau moderado-grave para os aspectos gravidez não planejada, dependência de álcool e/ou drogas, e presença de distúrbios de fala, linguagem e/ou audição em familiares, pais separados, pais ausentes e perda de parentes. Contudo essa diferença não foi estatisticamente significante. Para o aspecto distúrbios psicológicos, houve predomínio para o grupo de grau leve com diferença estatisticamente significante. Conclusão: os aspectos familiares estudados parecem não ter relação direta com a gravidade do desvio fonológico, com exceção de distúrbios psicológicos. Contudo, a investigação de aspectos familiares em crianças com desvio fonológico não deixa de ser importante para conduzir melhor o tratamento.

DESCRITORES: Crianças; Fala; Distúrbios da fala; Relações Familiares

(1) Fonoaudióloga; Professora do Curso de Fonoaudiologia da Universidade Federal de Santa Maria, UFSM, Santa Maria, RS; Mestre em Distúrbios da Comunicação Humana.

(2) Fonoaudióloga; Bolsista CAPES (Coordenação de Aperfeiçoamento de Pessoal de Nível Superior); Mestranda em Distúrbios da Comunicação Humana na Universidade Federal de Santa Maria.

(3) Fonoaudióloga; Professora do Curso de Fonoaudiologia e do Mestrado em Distúrbios da Comunicação Humana da Universidade Federal de Santa Maria, UFSM, Santa Maria, RS; Doutora em Lingüística Aplicada pela Pontifícia Universidade Católica do Rio Grande do Sul.

(4) Fonoaudióloga; Professora do Curso de Fonoaudiologia e do Mestrado em Distúrbios da Comunicação Humana da Universidade Federal de Santa Maria, UFSM, Santa Maria, RS; Doutora em Linguística Aplicada pela Pontifícia Universidade Católica do Rio Grande do Sul.

Conflito de interesses: inexistente

\section{INTRODUÇÃO}

A partir da idade de 4 anos a maioria das crianças possui seu sistema fonológico completo, porém, há algumas crianças que apresentam alterações de fala que persistem além dessa idade, isto é, têm dificuldades em organizar o sistema de sons de sua língua. Tais crianças não apresentam fatores orgânicos identificáveis (deficiência auditiva e anormalidades anatômicas e funcionais) que possam interferir em sua fala, o que caracteriza o desvio fonológico ${ }^{1}$.

$\mathrm{Na}$ prática clínica, observa-se que a inteligibilidade de fala e a gravidade do desvio fonológico não comprometem da mesma maneira todas as crianças. Assim, tanto a gravidade como a 
inteligibilidade de fala apresentam-se em graus variados nos desvios fonológicos ${ }^{2-7}$.

Uma forma bastante citada na literatura para classificar a gravidade do desvio fonológico é o Percentual de Consoantes Corretas $-\mathrm{PCC}^{8}$, medida que expressa a porcentagem de sons consonantais produzidos corretamente numa amostra de fala espontânea. O índice do PCC é obtido pela divisão do número de consoantes produzidas corretamente pelo número total de consoantes produzidas na amostra, multiplicado por cem. A partir desse cálculo, o desvio fonológico pode ser classificado em quatro graus de gravidade: leve (85 a 100\%); leve-moderado (65 a 85\%); moderado-grave (50 a $65 \%)$ e grave $(<50 \%)$. Outras maneiras de se calcular a gravidade são descritas na literatura ${ }^{3,9}$, porém a mais utilizada em pesquisas nacionais $\mathrm{e}$ internacionais é o PCC.

A etiologia do desvio fonológico é desconhecida, embora haja diversos trabalhos apresentando possíveis fatores influentes, tais como: sexo, idade $^{10}$, otites, alterações respiratórias ${ }^{11,12} e$, principalmente, o núcleo familiar ${ }^{13-19}$.

Além disso, fatores psicossociais, como baixo nível educacional dos pais, distúrbios psiquiátricos, paternidade precoce, famílias incompletas ou com graves problemas de relacionamento, são apontados como fatores de risco para problemas no desenvolvimento da linguagem e da fala ${ }^{20}$. Ainda, resultados de um estudo ${ }^{21}$ sugerem haver uma base familial nos desvios fonológicos graves.

Considerando tais estudos e a importância de investigar os aspectos familiares em crianças com desvio fonológico para conduzir melhor o tratamento, este trabalho teve como objetivo analisar a relação entre os fatores familiares e as diferentes gravidades do desvio fonológico.

\section{MÉTODO}

Esta pesquisa é de caráter transversal, exploratória e do tipo quantitativa. Foram analisadas as entrevistas iniciais de 152 crianças com desvio fonológico que faziam parte do banco de dados de um projeto de pesquisa. Os sujeitos eram de ambos os sexos, com idades entre 4:0 e 8:0 anos, e não haviam recebido tratamento fonoaudiológico anterior.

As entrevistas iniciais analisadas haviam sido realizadas com os responsáveis pelas crianças, por meio de protocolo específico, que compreende questões referentes à: história gestacional (gravidez, parto, condições do recém-nascido); alimentação; desenvolvimento psicomotor; má formação esquelética ou problema no desenvolvimento da dentição; qualidade do sono; controle de esfíncteres; desenvolvimento da linguagem; sexualidade (p.e. se a criança já demonstrou curiosidade sexual) escolaridade; características pessoais da criança (p.e. extrovertido, tímido), e atividades diárias; antecedentes fisiopatológicos (p.e. sarampo, rubéola, varíola); antecedentes patológicos familiares (doenças infecto-contagiosas da mãe durante a gravidez, transtornos emocionais, transtornos neurológicos, problemas de vias aéreas superiores, dificuldades de aprendizagem, trocas na fala, gagueira, demora para início da fala, alcoolismo, uso de drogas, fumo e consanguinidade) e, por fim, relacionamento familiar. O protocolo, em anexo, é composto por respostas abertas e fechadas.

Após a realização da anamnese, as crianças foram submetidas a uma série de avaliações para confirmar o diagnóstico de desvio fonológico. Foram avaliados os seguintes aspectos: linguagem (por meio de narração e conversa espontânea, utilizando uma sequência lógica de cinco fatos sobre o tema "aniversário"), fonológica, exame articulatório, sistema estomatognático, discriminação auditiva e vocabulário. Também foram realizados exames complementares (otorrinolaringológico, audiológico e neurológico).

Os dados da fala foram obtidos por meio da nomeação espontânea das figuras que compõe o instrumento de Avaliação Fonológica da Criança $\mathrm{AFC}^{22}$, a qual foi gravada e, posteriormente, transcrita foneticamente. Em seguida, foi realizada a análise contrastiva, com o objetivo de estabelecer o sistema fonológico da criança e, por último, calculado o Percentual de Consoantes Corretas - Revisado (PCC-R) ${ }^{9}$. Para a classificação dos graus de desvio fonológico seguiram-se os índices estabelecidos em um estudo ${ }^{8}$ que classifica o desvio em leve (86 a 100\%); leve-moderado (66 a 85\%); moderado-grave (51 a $65 \%)$; e grave $(<50 \%)$.

Como critérios de inclusão, o protocolo da entrevista inicial deveria estar completamente respondido e a criança apresentar alteração apenas na avaliação fonológica. Dos 209 sujeitos pertencentes ao banco de dados, apenas 152 preencheram tais critérios.

Nesta pesquisa, os fatores familiares investigados por meio do protocolo para fins de análise foram: gravidez não planejada; dependência de álcool e/ou drogas; distúrbios de fala, linguagem e audição; distúrbios psicológicos diagnosticados em familiares próximos; pais separados; pai ausente e perda de parentes próximos.

Os dados coletados pertencem a um projeto devidamente registrado e aprovado no Comitê de Ética em Pesquisa (CEP) sob número 052/04. Os responsáveis pelas crianças assinaram o Termo de 
Consentimento Livre e Esclarecido, autorizando a participação na pesquisa e posterior publicação dos resultados.

Para verificar a relação entre os aspectos familiares com as diferentes gravidades do desvio fonológico, os dados foram tabulados e submetidos a tratamento estatístico, utilizando o teste de Associação Qui-Quadrado e quando verificada diferença estatisticamente significante complementado pela Análise de Resíduos Ajustados. O nível de significância adotado foi de $5 \%(p<0,05)$.

\section{RESULTADOS}

Os resultados obtidos na Tabela 1 referem-se às frequências dos fatores familiares relatados na amostra. Verifica-se que gravidez não planejada apresentou maior ocorrência $(49,34 \%)$, seguida de distúrbios de fala, linguagem e/ou audição (38,82\%), dependência de álcool e/ou drogas $(23,03 \%)$ e pais separados (18,42\%). Já as frequências mais baixas foram observadas para perda de parentes $(3,95 \%)$, seguida de distúrbios psicológicos $(9,87 \%)$ e pais ausentes (15,79\%). Importante destacar que para todos os aspectos investigados, com exceção de gravidez não planejada, houve diferença estatisticamente significativa.

Na Figura 1 é apresentada a distribuição da gravidade do desvio fonológico, segundo o número de fatores familiares relatados na amostra. Verificase que em 41 casos (28,86\%) não houve nenhum aspecto familiar relatado. Ainda os graus leve e grave obtiveram o maior percentual de crianças que não apresentaram nenhum fator familiar $(32,65 \%$ e $33,33 \%$, respectivamente), enquanto que os graus leve-moderado e moderado-grave obtiveram o maior percentual de crianças que apresentaram mais de três aspectos familiares $(25,37 \%$ e $37,50 \%$, respectivamenteConvém ressaltar que não houve diferença estatística.

A Figura 2 compara a frequência dos fatores familiares nos diferentes graus do desvio fonológico. Observa-se que, no que diz respeito ao aspecto gravidez não planejada, houve ocorrência em todos os graus de desvio fonológico, sendo maior para o grau moderado-grave $(66,67 \%)$, porém não houve diferença estatisticamente significante.

Quanto ao aspecto dependência de álcool e/ou drogas, observa-se ocorrência em todos os graus de desvio fonológico, sendo maior para o grau moderado-grave $(33,33 \%)$, porém esta diferença não foi estatisticamente significante.

No que se refere aos antecedentes familiares com distúrbios de fala, linguagem e/ou audição, verifica-se maior percentual para o grau moderadograve $(50 \%)$, porém, não houve diferença estatisticamente significante

Os resultados referentes à presença de distúrbios psicológicos mostram associação estatisticamente

Tabela 1 - Relação dos fatores familiares com o desvio fonológico

\begin{tabular}{lccccc}
\hline Fatores Familiares & Presente $(\mathbf{n})$ & $\%$ & Ausente $(\mathbf{n})$ & $\%$ & $\mathbf{p}$ \\
\hline Gravidez não planejada & 75 & 49,34 & 77 & 50,66 & 0,818 \\
Dependência de álcool e/ou drogas & 35 & 23,03 & 117 & 76,97 & $<0,001$ \\
Distúrbio de fala, linguagem e/ou audição & 59 & 38,82 & 93 & 61,18 & $<0,001$ \\
Distúrbios psicológicos & 15 & 9,87 & 137 & 90,13 & $<0,001$ \\
Pais separados & 28 & 18,42 & 124 & 81,58 & $<0,001$ \\
Pais ausentes & 24 & 15,79 & 128 & 84,21 & $<0,001$ \\
Perda de parentes & 6 & 3,95 & 146 & 96,05 & $<0,001$ \\
\hline
\end{tabular}

\begin{tabular}{|c|c|c|c|c|c|c|c|c|c|}
\hline \multirow{3}{*}{$\begin{array}{l}\text { Número de } \\
\text { fatores } \\
\text { familiares }\end{array}$} & \multicolumn{8}{|c|}{ Gravidade } & \multirow{3}{*}{ p } \\
\hline & \multicolumn{2}{|c|}{ DL } & \multicolumn{2}{|c|}{ DLM } & \multicolumn{2}{|c|}{ DMG } & \multicolumn{2}{|c|}{$D G$} & \\
\hline & $\mathrm{n}=49$ & $\%$ & $\mathrm{n}=67$ & $\%$ & $\mathrm{n}=24$ & $\%$ & $\mathrm{n}=12$ & $\%$ & \\
\hline Nenhum & 16 & 32,65 & 19 & 28,36 & 2 & 8,33 & 4 & 33,33 & \\
\hline Apenas 1 & 16 & 32,65 & 15 & 22,39 & 6 & 25,00 & 4 & 33,33 & 264 \\
\hline Apenas 2 & 8 & 16,33 & 16 & 23,88 & 7 & 29,17 & 3 & 25,00 & 0,204 \\
\hline Mais que 3 & 9 & 18,37 & 17 & 25,37 & 9 & 37,50 & 1 & 8,34 & \\
\hline
\end{tabular}

Figura 1 - Distribuição do número de fatores familiares segundo a gravidade do desvio fonológico 


\begin{tabular}{|c|c|c|c|c|c|c|c|c|c|c|}
\hline \multirow{2}{*}{\multicolumn{2}{|c|}{ Fatores Familiares }} & \multicolumn{2}{|c|}{ DL } & \multicolumn{2}{|c|}{ DML } & \multicolumn{2}{|c|}{ DMG } & \multicolumn{2}{|c|}{ DG } & \multirow{2}{*}{$\mathbf{p}$} \\
\hline & & $\mathrm{n}$ & $\%$ & $n$ & $\%$ & $n$ & $\%$ & $\mathrm{n}$ & $\%$ & \\
\hline \multirow{2}{*}{$\begin{array}{l}\text { Gravidez não } \\
\text { planejada }\end{array}$} & Sim & 24 & 48,98 & 31 & 46,27 & 16 & 66,67 & 4 & 33,33 & \multirow{2}{*}{0,220} \\
\hline & Não & 25 & 51,02 & 36 & 53,73 & 8 & 33,33 & 8 & 66,67 & \\
\hline \multirow{2}{*}{$\begin{array}{l}\text { Dependência de } \\
\text { álcool e/ou } \\
\text { drogas }\end{array}$} & Sim & 10 & 20,41 & 15 & 22,39 & 8 & 33,33 & 2 & 16,67 & \multirow[b]{2}{*}{0,613} \\
\hline & Não & 39 & 79,59 & 52 & 77,61 & 16 & 66,67 & 10 & 83,33 & \\
\hline \multirow{2}{*}{$\begin{array}{l}\text { Distúrbio de } \\
\text { fala, linguagem } \\
\text { e/ou audição }\end{array}$} & Sim & 17 & 34,69 & 27 & 40,30 & 12 & 50,00 & 3 & 25,00 & \multirow[b]{2}{*}{0,482} \\
\hline & Não & 32 & 65,31 & 40 & 59,70 & 12 & 50,00 & 9 & 75,00 & \\
\hline \multirow{2}{*}{$\begin{array}{l}\text { Distúrbios } \\
\text { psicológicos }\end{array}$} & Sim & 48 & 97,96 & 6 & 8,96 & 6 & 25,00 & 2 & 16,67 & \multirow{2}{*}{0,013} \\
\hline & Não & 1 & 2,04 & 61 & 91,04 & 18 & 75,00 & 10 & 83,33 & \\
\hline \multirow[t]{2}{*}{ Pais separados } & Sim & 6 & 12,24 & 14 & 20,90 & 7 & 29,17 & 1 & 8,33 & \multirow{2}{*}{0,269} \\
\hline & Não & 43 & 87,76 & 53 & 79,10 & 17 & 70,83 & 11 & 91,67 & \\
\hline \multirow[t]{2}{*}{ Pais ausentes } & Sim & 7 & 14,29 & 11 & 16,42 & 5 & 20,83 & 1 & 8,33 & \multirow{2}{*}{0,828} \\
\hline & Não & 42 & 85,71 & 56 & 83,58 & 19 & 79,17 & 11 & 91,67 & \\
\hline \multirow{2}{*}{$\begin{array}{l}\text { Perda de } \\
\text { parentes }\end{array}$} & Sim & 1 & 2,04 & 3 & 4,48 & 2 & 8,33 & 0 & 0,00 & \multirow{2}{*}{0,495} \\
\hline & Não & 48 & 97,96 & 64 & 95,52 & 22 & 91,67 & 12 & 100,00 & \\
\hline
\end{tabular}

Figura 2 - Relação dos fatores familiares com a gravidade do desvio fonológico

significante com a gravidade do desvio. Além disso, observa-se associação positiva entre o fator familiar distúrbios psicológicos e o grau leve e associação negativa deste fator com o grau leve-moderado. Tais achados permitem afirmar que o fator familiar distúrbios psicológicos ocorre com maior frequencia no desvio Leve.

Quando a distribuição entre pais separados e pais ausentes foi comparada segundo a gravidade do desvio fonológico, observou-se que ambas foram mais representativas para o grau moderado-grave $(29,17 \%$ e $20,83 \%$, respectivamente), apesar de não haver diferença estatisticamente significante.

No que se refere à perda de parentes, foram poucos os casos registrados, sendo que o grupo do desvio fonológico grave não registrou nenhum caso. Ainda, o maior percentual foi verificado para o grau moderado-grave (8,33\%). No entanto, não houve diferença estatisticamente significante.

\section{DISCUSSÃO}

A qualidade das relações afetivas familiares desempenha um importante papel no desenvolvimento da linguagem, sendo a separação parental, desentendimento crônico, alcoolismo, doença crônica de um genitor, casais incompletos (p.e. mãe solteira) e óbito fatores de risco para que ocorra um distúrbio no desenvolvimento lingüístico ${ }^{23}$. Apesar de esses fatores apontarem para uma relação com a linguagem, os achados deste estudo não são suficientes e específicos para afirmar que a questão familiar seja um agravante para a gravidade do desvio fonológico.

Os achados desta pesquisa, quanto à presença de distúrbios de fala, linguagem e/ou audição, concordam com outras já realizadas ${ }^{14,16}$, as quais referem que é comum em crianças com alteração de fala ou de linguagem observar a presença de antecedentes familiares de risco. Nesse sentido, as influências parentais e os antecedentes fisiopatológicos são apontados como importantes no desenvolvimento da linguagem e distúrbios da articulação ${ }^{24}$.

O percentual encontrado neste estudo $(38,82 \%)$ assemelha-se ao verificado na literatura ${ }^{13}$, em que os autores sugerem que crianças provenientes de famílias nas quais há relato de pessoas com alterações de fala e linguagem, durante a infância, diferem de crianças provenientes de famílias nas quais não foram encontradas tais alterações de habilidades de produção fala. O estudo mostra que $20 \%$ a $40 \%$ dos sujeitos com distúrbio de fala e linguagem possuem casos desses distúrbios na família.

Em outro estudo ${ }^{16}$, as autoras concluíram que existem fatores que indicam que o histórico de transtorno de fala e linguagem no núcleo familial está associado ao transtorno fonológico da criança e que tendo um controle sobre a história do desvio pode-se saber se a criança tem possibilidade de tê-lo ou não. Apesar da referida importância dos aspectos familiares, não há evidências de quais fatores ambientais ou genéticos seriam determinantes na 
geração dos desvios fonológicos ${ }^{21}$. De modo similar a esses estudos, não foram evidenciados fatores estatisticamente relevantes na geração do desvio fonológico, a partir dos resultados encontrados na presente pesquisa.

Um estudo recente ${ }^{19}$ teve como objetivo comparar a evolução terapêutica de dois irmãos com desvio fonológico com alterações semeIhantes, tratados individualmente, e verificou que um dos aspectos limitantes para o tratamento foi o fator ambiental, o qual dificultou a aquisição dos fonemas trabalhados, uma vez fora do contexto de terapia, o padrão fonológico alterado era reforçado entre os irmãos. As autoras concluíram que talvez seria mais eficaz se ambos fossem tratados conjuntamente para supressão do desvio.

Comparando a presença de distúrbios de fala, linguagem e/ou audição, nos diferentes graus de desvio fonológico, os achados do presente estudo não são suficientes para afirmar que tais fatores têm influência sobre a gravidade do desvio fonológico. Apenas a associação entre problemas psicológicos e desvio fonológico leve apresentou-se relevante estatisticamente. Uma hipótese, com base nesse dado, é que crianças com desvios fonológicos leves estejam prolongando a fase final de aquisição fonológica por demandas psicológicas geradas no contexto familiar.

Essa hipótese ganha força quando se sabe que as alterações de fala mais comuns nesse grupo são relacionadas à aquisição do tepe e de estruturas silábicas complexas como o onset complexo, aquisições características da etapa final da aquisição fonológica. Por outro lado, estudos do desenvolvimento infantil, em especial os realizados por Winni$\operatorname{cott}^{25}$, demonstram que há crianças que resistem em ultrapassar a fase da dependência relativa para a da independência das figuras parentais, e, em geral, este fato relaciona-se a aspectos psicológicos presentes no histórico familiar. Tal dependência relativa pode ser manifestada sintomaticamente pela detenção em uma etapa fonológica anterior à idade cronológica. No entanto, tais aspectos deverão sofrer maior investigação em estudos de coorte.

Estudos ${ }^{23,26}$ apontam o alcoolismo parental como um fator de risco que pode trazer prejuízos para o desenvolvimento infantil. Porém não foi encontrado nenhum estudo que correlacione o desvio fonológico com o alcoolismo parental. Tal correlação não se evidenciou nos resultados desta pesquisa.

\section{CONCLUSÃO}

Diante do exposto, podemos concluir que os aspectos familiares estudados parecem não ter relação direta com a gravidade do desvio fonológico, com exceção de distúrbios psicológicos nas crianças com desvio fonológico leve.

Há, no entanto, a necessidade de tais desvios serem investigados a partir de um acompanhamento longitudinal dos sujeitos, pois os familiares, quando citam algum fator possivelmente relevante, muitas vezes não possuem um diagnóstico sobre o mesmo.

De um modo geral, esta pesquisa empreendeu uma primeira aproximação do tema indicando caminhos a serem mais bem explorados, pois, mesmo sem haver uma relação causal entre desvio fonológico e aspectos familiares, esses aspectos podem, ao menos, estar alimentando a permanência do desvio fonológico quando incidem em condições psicológicas presentes no ambiente familiar, como sinalizou o resultado para o desvio fonológico de grau leve. 


\begin{abstract}
Purpose: to analyze the relationship among the familial factors and the different severities of phonological disorder. Method: we analyzed initial interviews that were carried out through standardized protocol that included questions regarding pregnancy history, family pathological history and family relationship of 152 children with phonological disorder, ages 4:0 to 8:0-year old. The familial factors investigated were: unplanned pregnancy; dependency (one of the parents and/or both) on alcohol and/ or drugs; speech, language and hearing disorders (shown by the parents and/or first-degree relatives); psychological disorders (shown by the parents); separated parents; father absence and loss of close relatives. Then, the severity of phonological disorder was calculated according to the Percentage of Correct Consonants and classified in four groups: mild $(n=49)$, mild-moderate $(n=67)$, moderatesevere $(n=24)$, severe $(n=12)$. Finally, data were tabulated and submitted to statistical treatment using the Fisher Exact Test, considering $p<0.05$. Results: we verified predominance of the group with moderate-severe disorder as for the aspects of unplanned pregnancy, dependency on alcohol and/ or drugs, speech language and/or hearing disorders in relatives, separated parents, absent parents and loss of relatives. However, this difference was not statistically significant. For the psychological disorders aspect, there was a predominance of the mild group with a statistically significant difference. Conclusion: the familial aspects studied seem not to have direct relationship with the severity of phonological disorder, except for the psychological disorders. However, the investigation of familial aspects in children with phonological disorder is important to better conduct the treatment.
\end{abstract}

KEYWORDS: Children; Speech; Speech Disorders; Family Relations

\section{REFERÊNCIAS}

1. Grunwell P. Os desvios fonológicos numa perspectiva lingüística. In: Yavas $M$. Desvios fonológicos em crianças: teoria, pesquisa e tratamento. Porto Alegre: Mercado Aberto; 1990. p. 53-77.

2. Wertzner HF, Amaro L, Teramoto S. Descritores da classificação da gravidade do distúrbio fonológico. Pró-Fono. 2004; 16(2):139-50.

3. Keske-Soares M, Blanco APF, Mota HB. O desvio fonológico caracterizado por índices de substituição e omissão. Rev Soc Bras Fonoaudiol. 2004; 9(1):30-6.

4. Viera MG, Mota HB, Keske-Soares M. Relação entre idade, grau de severidade do desvio fonológico e consciência fonológica. Rev Soc Bras Fonoaudiol. 2004; 9(3):144-50.

5. Whitehead RL, Schiavetti N, Mackenzie DJ, Metz DE. Intelligibility of speech produced during simultaneous communication. J Commun Dis. 2004; 37(3):241-53.

6. Flipsen Junior P, Hummer JB, Yost KM. Measuring severity of involvement in speech delay: segmental and whole-word measures. Am J Speech Lang Path. 2005; 14:298-312.

7. Donicht G, Pagliarin KC, Mota HB, Keske-Soares M. A inteligibilidade do desvio fonológico julgada por três grupos de julgadores. Pró-Fono. No prelo 2009.

8. Shriberg LD, Kwiatkowski J. Phonological disorders I: a diagnostic classification system. J Speech Hear Dis. 1982; 47:226-41.

9. Shriberg L, Austin D, Lewis B, McSweeny J, Wilson D. The percentage of consonants correct (PCC) metric: extensions and reliability data. J Speech Lang Hear Res. 1997; 40:708-22.

10. Wertzner HF, Oliveira MMF. Semelhanças entre os sujeitos com distúrbio fonológico. Pró-Fono. 2002; 14(2):143-52.

11. Wertzner HF, Pagan LO, Galea DES, Papp ACCS. Características fonológicas de crianças com transtorno fonológico com e sem histórico de otite média. Rev Soc Bras Fonoaudiol. 2007; 12:41-7.

12. Souza APR, Pergher GL, Pagliarin KC. Aspectos motores corporais e orais em um grupo de crianças com transtorno/atraso fonológico. Rev Soc Bras Fonoaudiol. No prelo 2010.

13. Lewis BA, Freebairn L. Residual effects of preschool phonology disorders in grade schools, adolescence, and adulthood. J Speech Hear Res. 1997; 35:819-31.

14. Lewis BA, Freebairn L, Hansen M, Taylor HG, lyengar S, Shriberg LD. Family pedigrees of children with suspected childhood apraxia of speech. J Comm Dis. 2004; 37:157-75. 
15. Kovas Y, Hayiou-Thomas ME, Oliver B, Dale PS, Bishop DV, Plomin R. Genetic influences in different aspects of language development: the etiology of language skills in 4.5-year-old twins. Child Dev. 2005; 76(3):632-51.

16. Wertzner HD, Papp ACCS. O aspecto familial e o transtorno fonológico. Pró-Fono. 2006; 18(2):151-60.

17. Lewis BA, Freebairn LA, Hansen AJ, Miscimarra L, lyengar SK, Taylor HG. Speech and language skills of parents of children with speech sound disorders. Am J Speech-Lang Path. 2007; 16:108-18.

18. Weber DE, Vares MA, Mota HE, Keske-Soares M. Desenvolvimento do sistema fonológico de gêmeos monozigóticos com desvio fonológico: correlação a fatores genéticos e ambientais. Rev CEFAC. 2007; 9(1):32-9.

19. Pagliarin KC, Keske-Soares M, Mota H. Terapia fonológica em irmãos com diferentes graus de gravidade do desvio fonológico. Rev CEFAC. 2009; 11(1):20-4.
20. Mysak ED. Patologias dos sistemas da fala. Identificação dos distúrbios da fala, princípios de exame e tratamento. 2. ed. São Paulo: Atheneu; 1998.

21. Lewis BA, Ekelman BL, Aram DM. A familial study of severe phonological disorders. J Speech Hear Res. 1989; 32:713-24.

22. Yavas M, Hernandorena CLM, Lamprecht RR. Avaliação fonológica da criança: reeducação e terapia. Porto Alegre: Artes Médicas; 2001.

23. Marcelli D. Manual de psicopatologia infantil de Ajuriaguerra. 5. ed. Porto Alegre: Artes Médicas; 1998.

24. Riper CV, Emerick L. Correção da linguagem: uma introdução à patologia da fala e à audiologia. Porto Alegre: Artes Médicas; 1997.

25. Winnicott D. Da pediatria à psicanálise:obras escolhidas. Rio de Janeiro: Imago; 2000.

26. Ajuriaguerra J, Marcelli D. Manual de psicopatologia infantil. 2. ed. Porto Alegre: Artes Médicas; 1986.

Endereço para correspondência:

Karina Carlesso Pagliarin

Rua Coronel Scherer, 09

São Pedro do Sul - RS

CEP: 97400-000

E-mail: karinap_fono@yahoo.com.br 


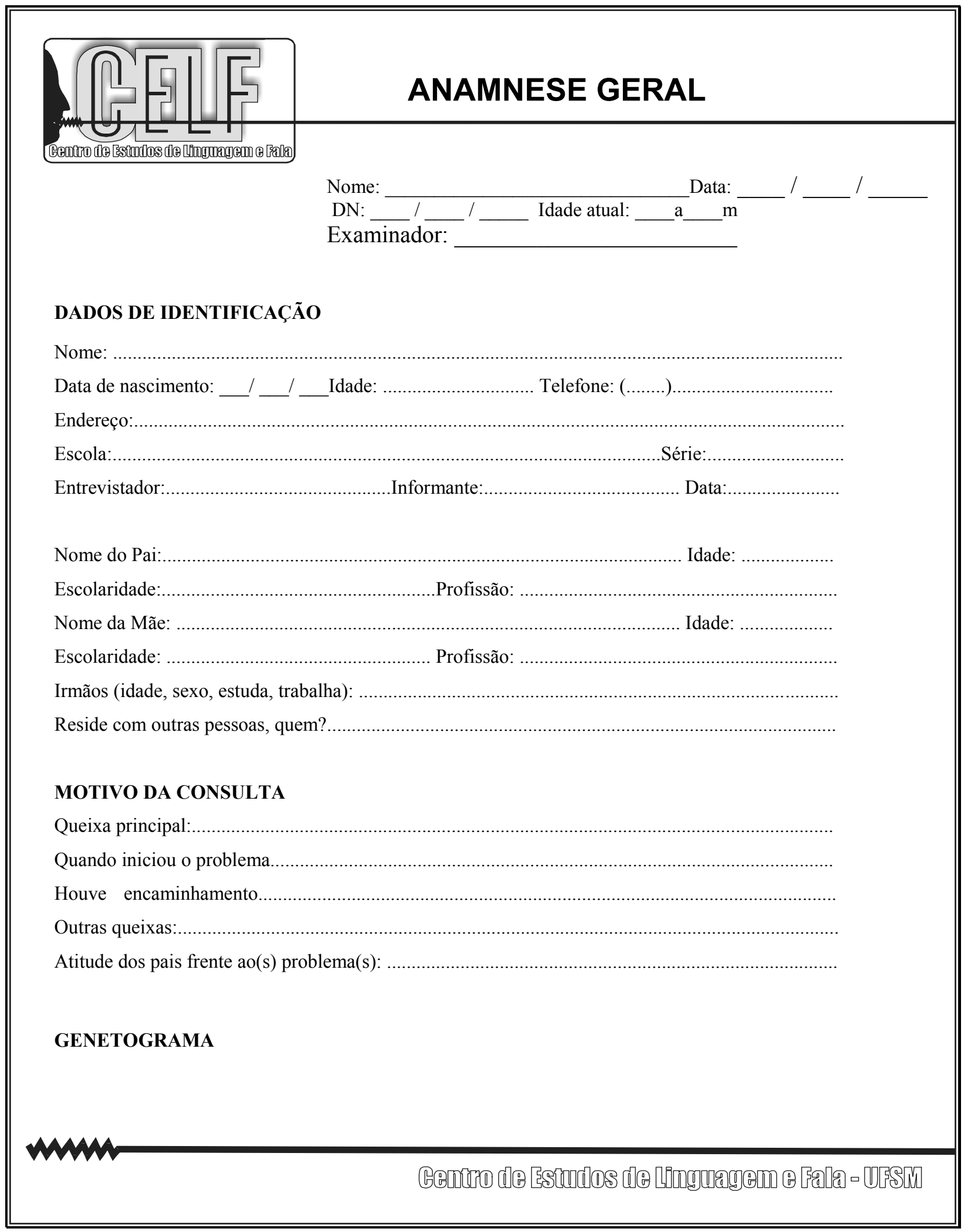




\section{GRAVIDEZ:}

Idade da mãe na gravidez: A gravidez foi planejada: $\square \operatorname{sim} \quad \square$ não

Houve ameaça de aborto: $\square$ leucorragias $\square$ hemorragia

O médico exigiu repouso: $\square \operatorname{sim} \square$ não

O período de gravidez foi acompanhado de: $\square$ vômitos $\quad \square$ enjôos $\quad \square$ nervosismo

Como:.

Houve tratamento pré-natal: $\square$ sim $\square$ não $\quad$ Fator Rh (mãe):

exame de sangue: ex. de urina: radiografia até o $3^{\circ}$ mês:

Doenças: $\square$ citomegalovírus $\square$ toxoplasmose $\square$ diabete gestacional $\square$ sífilis $\quad \square$ rubéola $\quad \square$ gripes $\square$ hipertensão $\quad \square$ hipertireoidismo $\square$ doenças cardíacas $\square$ febre alta, quanto:.........

$\square$ hepatite $\square$ fumo $\quad \square$ álcool $\square$ drogas

$\square$ tombos, características:

Medicamentos durante a gravidez: $\square$ não $\square$ sim, quais:

Qual a expectativa da família quanto ao bebê ?....

Observações:

\section{PARTO}

$\square$ a termo $\square$ pré-termo $\square$ pós-termo $\quad$ Meses de gestação:

Rompimento anterior da bolsa ? $\square \operatorname{sim} \square$ não Quando ?

O parto foi: $\square$ na maternidade $\square$ em casa atendido por:

$\square$ normal $\square$ a vácuo $\quad \square$ fórceps $\quad \square$ cesárea $\quad \square$ demorado ................. $\square$ rápido ........

$\square$ anestesia Qual?

Obs:

\section{CONDIÇÕES DO RECÉM NASCIDO}

Peso:

Comprimento: Posição no nascimento:

A cabeça pareceu: $\square$ pequena $\square$ grande $\quad \square$ chorou logo $\square$ precisou de oxigênio

Cor da criança ao nascer: $\square$ natural $\quad \square$ roxo $\quad \square$ pálido $\quad \square$ icterícia $\quad \square$ fez banho de luz $\square$ apresentou alguma má formação $\square$ a posição do cordão umbilical era adequada

Primeiras reações dos familiares quanto ao bebê:

Obs: 


\section{ALIMENTAÇÃO}

$\square$ amamentação natural Até quando:

Como e porque ocorreu o desmame:

Teve dificuldade de sucção ou deglutição:

$\square$ mamadeira Quando parou?

$\square$ chupeta Quando parou?

Alimentação sólida: quando iniciou?. consistência:

Atualmente come bem: $\square$ sim $\square$ não

É forçado a se alimentar: $\square$ não $\square$ sim, como:

Escolhe alimentos: $\square$ não $\square$ sim, quais:

É sujeito a: $\square$ vômitos

diarréia

$\square$ constipação

Quando e como ocorre?

Obs:

\section{DENTIÇÃO}

Iniciou aos .meses. Alguma particularidade:

\section{SONO}

Como era o sono quando bebê:

Sono atual: $\square$ tranqüilo $\square$ agitado $\square$ muda muito de lugar $\quad \square$ fala $\quad \square$ grita $\square$ bate-se na cama

$\square$ sua muito $\quad \square$ terror noturno $\square$ sonambulismo $\square$ mexe braços e pernas $\quad \square$ acorda muito

$\square$ range os dentes $\square$ dorme com a boca aberta $\square$ ronca $\square$ baba

Dorme no quarto com os pais: $\square$ sim $\square$ não Até quando dormiu:

Como foi feita a mudança:

Como a criança se sentiu:

Tem cama individual: $\square$ sim $\square$ não, divide com quem:

Hora de deitar:.

$\square$ sozinho $\square$ auxílio $\quad$ Hora de levantar:

Obs:

\section{DESENVOLVIMENTO PSICOMOTOR}

Sustentou a cabeça:

Sentou:

Engatinhou:

Ficou em pé: 
Se engatinhou, como foi?

Se não, por quê?.

Como foi o início da marcha?

$\square$ apresentou alguma dificuldade motora

$\square$ foi obrigado a usar a mão direita

Obs:

\section{CONTROLE ESFINCTERIANO}

Evacuação: Como foi o controle?.

Urina: Como foi o controle?

\section{DESENVOLVIMENTO DA LINGUAGEM (colocar idade e exemplos)}

Período de balbucio:

Primeiras palavras:

Frases simples:

$\square$ uso do pronome 'eu': $\square$ uso de gestos para se comunicar $\quad \square$ compreende ordens $\square$ apresentou gagueira, quando:. .reação dos familiares:

Quais trocas nota na fala:

As pessoas da família entendem a fala: E as outras pessoas:

Como vê o problema de fala:

Como reage em relação a isso:

Como explica o problema de fala para seu filho:

Obs:

\section{SEXUALIDADE}

Demonstrou curiosidade sexual: De que forma:

Atitude tomada:

Desde quando e como é realizada a educação sexual:

Obs: 


\section{ESCOLARIDADE}

Escolas anteriores / séries / desempenhos:

Desempenho atual:

Turno:

\section{RELACIONAMENTO}

Entre os pais:

Entre a mãe e a criança:

Entre o pai e a criança:

Entre os irmãos e a criança:

Entre os pais e os demais filhos:

Entre os avós e os pais da criança:

Relaciona-se melhor com: $\square$ mãe $\quad \square$ pai $\quad \square$ outra

Na escola:

Obs:

\section{CARACTERÍSTICAS PESSOAIS}

$\square$ faz amizade com facilidade $\quad \square$ convida amigos $\quad \square$ vai a festas e passeios

Prefere brincar com crianças: $\square$ maiores $\square$ menores

$\square$ introvertido $\quad \square$ extrovertido $\quad \square$ calmo $\quad \square$ agressivo $\quad \square$ dependente $\quad \square$ independente
$\square$ humor variável $\square$ observador $\quad \square$ alegre $\quad \square$ retraído $\quad \square$ crises de birra $\square$ rói unhas
$\square$ puxa os cabelos $\quad \square$ chupa dedo $\quad \square$ morde os lábios $\quad \square$ chora ou ri sem motivo

Como reage quando contrariado:

Atitude dos pais:.

Como reage em situações novas?

Obs:

\section{ATIVIDADES DIÁRIAS}

O que faz nos momentos livres?...

Tem atividade além da escola? $\square$ não $\square$ sim, quais?

Lugar em que brinca:. 
Com quem brinca:

Toma banho só? $\square$ sim, desde quando? não, por quê?

$\square$ escova os dentes $\quad \square$ penteia os cabelos $\quad \square$ veste-se $\quad \square$ abotoa a roupa $\quad \square$ amarra os sapatos

$\square$ cuida do material escolar, desde quando?

$\square$ estas atitudes são executadas espontaneamente ou $\square$ por imposição de um adulto

Obs:

\section{ANTECEDENTES FISIOPATOLÓGICOS}

\begin{tabular}{|c|c|c|}
\hline sarampo & $\square$ rubéola & $\square$ varíola \\
\hline
\end{tabular}

Vias aéreas superiores: $\square$ asma $\square$ bronquite $\square$ renite $\square$ sinusite $\square$ faringite/amigdalites frequentes

$\square$ hipertrofia de amígdalas $\quad \square$ hipertrofia de adenoides

Problemas auditivos: $\square$ dores de ouvido frequentes, quantas vezes/idades.

$\square$ otites, quantas vezes/idades.

$\square$ cirurgias, quais: quando: anestesia:

$\square$ vacinas em dia $\quad \square$ BCG $\quad \square$ outras

Está sendo medicado? $\square$ não $\square$ sim, qual?

Por indicação de quem?

Acha que enxerga bem: Acha que ouve bem:

Já procurou psiquiatra ou psicólogo? $\square$ não $\square \operatorname{sim}$, porque:

Já procurou outros profissionais? $\square$ não $\square$ sim, quais?

Obs:

\section{ANTECEDENTES PATOLÓGICOS FAMILIARES}

(Em caso de respostas afirmativas especifique o parentesco com a criança)

Doenças Infecto-Contagiosas:

Transtornos emocionais: $\square$ nervosismo depressão: $\checkmark$ outro

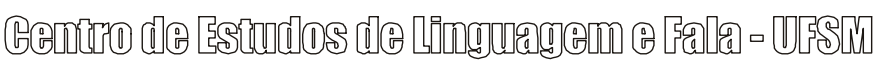


Transtornos neurológicos: $\square$ epilepsia:

convulsão: síndrome:

$\square$ portador de necessidades especiais (def. auditiva, def. mental, def. física):

Problemas de visão:

Problemas de vias aéreas superiores:

$\square$ trocas na fala:

$\square$ gagueira:

demorou a falar

Dificuldade na escola (em que área)

$\square$ álcool (hábito): drogas: fumo:

$\square$ consanguinidade: outros.

$\square$ internação, por quê?

Obs:

CONDUTA FONOAUDIOLÓGICA

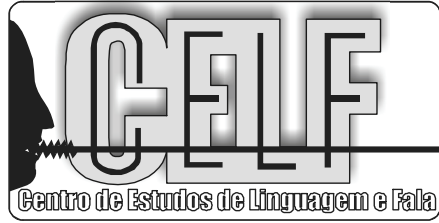

